\title{
Computationally efficient phase-field models with interface kinetics
}

\author{
Kalin Vetsigian and Nigel Goldenfeld \\ Department of Physics, \\ University of Illinois at Urbana-Champaign \\ 1110 West Green Street \\ Urbana, IL, 61801-3080
}

(Dated: July 1, 2018)

\begin{abstract}
We present a new phase-field model of solidification which allows efficient computations in the regime when interface kinetic effects dominate over capillary effects. The asymptotic analysis required to relate the parameters in the phase-field with those of the original sharp interface model is straightforward, and the resultant phase-field model can be used for a wide range of material parameters.
\end{abstract}

PACS numbers: $68.70 .+\mathrm{w}, 81.30 . \mathrm{Fb}, 47.54 .+\mathrm{r}$

Phase-field model techniques have become increasingly recognized as the tool of choice for solving moving free boundary (sharp-interface) problems, and in particular solidification processes [1, 2, 3, 44]. Following early work that related the phase-field models to the original sharp interface model in the limit of zero interface width [5], Karma and Rappel showed that calculations could be performed when the interface width is of the order of the capillary length [6, 7], even scaling with the dendritic tip radius at low undercooling. This work provided a particular relation between the parameters of the original sharp interface model and the phase-field model, and has been the starting point for accurate computations of solidification in the important regime when capillary effects dominate interface kinetics [8].

Recently however, there has been a growing interest in the opposite regime where interface kinetics are dominant. This interest is stimulated by experimental observation of the puzzling morphological transition of the solidification front of Ni at high undercooling [9, 10, 11, 12].

The purpose of this paper is to present a modification of the phase-field model of solidification so as to enable efficient computations in the regime when interface kinetics are the dominant factor. The modification allows one to use an interface thickness many times larger than the capillary length. The methodology for performing the asymptotic analysis is different and much easier to perform systematically to high order than the asymptotic matching employed in all previous analyses, and is capable of being used in other free boundary problems.

Sharp interface model:- The symmetric model for the solidification of a pure melt from the liquid (L) phase to the solid phase $(\mathrm{S})$ is defined by the equations:

$$
\begin{array}{r}
\partial_{t} u=D \nabla^{2} u \\
\left.\partial_{n} u\right|_{S}-\left.\partial_{n} u\right|_{L}=V / D \\
u_{i}+d_{0} k=-\mathcal{B}(V) .
\end{array}
$$

Here $u=\left(T-T_{M}\right) c / L$ is the dimensionless temperature and $u_{i}$ is its value at the solidification front, with $T$ being the temperature in the liquid or solid, $T_{M}$ being the melting temperature of a planar interface, $c$ being the specific heat and $L$ being the latent heat of fusion per unit volume. The curvature of the solidification front is given by $k$ and the capillary length is $d_{0} . D$ is thermal diffusivity (assumed here to be the same in both phases), and $V$ is the normal velocity of the front. Equation (2) expresses heat conservation at the interface, and equation (31) is a modified Gibbs-Thomson condition, which is a statement of local equilibrium at the interface with the attachment kinetics included through the term $\mathcal{B}(V)$. Traditionally, a linear kinetic undercooling $\mathcal{B}(V)=\beta V$ is used. It should be stressed, however, that the linearity of $u_{i}$ with respect to velocity is a purely phenomenological assumption, and molecular dynamics simulations [13, 14 suggest that there are substantial deviations from it at large undercooling. In this paper we are interested in materials with large dimensionless parameter $\tilde{\beta} \equiv \beta D / d_{0}$. This constant is a measure of the importance of interface kinetics for a given material. It takes very different values for different materials, and for $\mathrm{Ni}$ it is estimated from molecular dynamics simulations to be as high as 90 15].

The phase-field model:- The phase-field equations can generally be written in the form:

$$
\begin{aligned}
\tau \partial_{t} \psi & =W^{2} \nabla^{2} \psi-f_{\psi}(\psi)-\lambda u g_{\psi}(\psi) \\
\partial_{t} u & =D \nabla^{2} u+\frac{1}{2} \partial_{t} h(\psi) .
\end{aligned}
$$

Here $\psi$ represents the phase-field, $f(\psi)$ is a double well potential, $g(\psi)$ shifts the relative height of the two minima making one of the phases metastable for $u \neq 0$. Note that we have used the subscript notation to denote differentiation: hence $g_{\psi}(\psi)$ means $\partial g / \partial \psi$. The sharp interface boundary is recovered as the locus of points where $\psi=0$, and we are interested in the behavior of the phase-field equations as the phase-field interface width $W$ and relaxation time $\tau$ tend towards 0 . In order to solve the desired sharp interface model, we need to ascertain what phase-field parameters $\{\tau, W, \lambda, f(\psi), g(\psi)\}$ should be used given the values of $\left\{D, d_{0}, \beta\right.$ or $\left.\mathcal{B}(V)\right\}$.

Asymptotic analysis:- The limit of small $W$ is a singular one because $W$ multiplies a highest order derivative. All previous works use asymptotic matching to deal with the singularity. Here we will demonstrate a simpler ap- 
proach based on the fact that the equation for $u$ is linear so that it can be trivially solved in terms of $\psi$. Following Karma and Rappel, the analysis we give here focuses on the coordinate perpendicular to the interface, $r$; the effect of the transverse dimensions is incorporated by curvature dependent corrections, just as in their work.

Requiring that $u$ is finite at $r \rightarrow \pm \infty$ and requiring that $\psi(r) \rightarrow \mp 1$ sufficiently fast one obtains

$$
\begin{aligned}
u(\xi) & =u_{0}+\frac{1}{2} p e^{-(p+q) \xi} \int_{-m \infty}^{\xi} e^{(p+q) \eta} h(\psi(\eta)) d \eta \\
& =u_{0}+\frac{1}{2} p\left(\hat{u}+\frac{1}{p+q}\right),
\end{aligned}
$$

where

$$
q \equiv k W, \quad v \equiv \frac{V \tau}{W} \ll 1, \quad p \equiv \frac{V W}{D}, \quad \xi \equiv r / W,
$$

$m \equiv \operatorname{sgn}(p+q)$, and the last line defines $\hat{u}$. The dependence on $p+q$ expresses the singular nature of the problem with respect to this parameter. From $u(\xi)$ one can compute the outer limit $u_{\text {out }}(r)$ and thus obtain $u_{i}=u_{\text {out }}(0)=u_{0}+1 / 2 p /(p+q)$.

Despite the singularity, if we are interested only in the profile of $u$ near the interface one can expand in powers of $p$ and $p+q$ and to first order obtain

$$
u(\xi)=u_{i}+\frac{1}{2} p \int_{-\infty}^{\xi}(h(\psi(\eta))-1) d \eta+O(p(p+q)) .
$$

Using this expression and substituting it back in the equation for $\psi$ we get an equation for $\psi$ which can be solved using regular perturbation theory. In this way we recover the standard asymptotic result of Karma and Rappel:

$$
W(\lambda)=\lambda \frac{d_{o}}{a_{1}}, \quad \tau(\lambda)=\lambda^{2}\left(\frac{\beta D}{d_{0}}+a_{2} \lambda\right) \frac{d_{0}^{2}}{a_{1}^{2} D},
$$

where $a_{1}$ and $a_{2}$ are constants depending on $f(\psi)$ and $g(\psi)$. Notice also that since $\psi$ is monotonic one can compute the distance from the interface $\xi$ from $\psi$ and in this way write to order $p$

$$
u(\xi)=u_{i}+\frac{1}{2} p F_{1}(\psi ;\{\psi(\xi)\}),
$$

where $F_{1}$ is defined as the integral in equation (9).

From (10) follows that with the functions $f(\cdot), g(\cdot)$, and $h(\cdot)$ fixed, $\lambda$ is the only free parameter.

Computational complexity:- We now examine how the computation time $t_{c}$ for the phase-field model scales with the free parameter $\lambda$ in a discretized calculation with adaptive mesh refinement and uniform grid elements in $d$ dimensions. Clearly, $t_{c}$ depends on the width of the phase-field boundary layer $\tilde{W}$, the space resolution $\Delta x$, and the time step $\Delta t$. The inverse computation time scales as: $(\Delta t / t)(\Delta x / \tilde{W})(\Delta x / L)^{d-1}$ where $L^{d-1}$ is the order of the surface area and $t$ is the maximum time one wishes to evolve the system. For a spatially explicit numerical scheme $\Delta t \leq \frac{1}{2} \tau(\Delta x / W)^{2} / \lambda$. The factor of $\lambda$ is included to guarantee accuracy in the presence of the term $\lambda u g_{\psi}$. Collecting terms, and using $\tilde{W} \approx W \propto \lambda d_{0}$ and $\tau \propto \lambda^{2}$ we obtain

$$
t_{c}^{-1} \propto \lambda^{d}\left(\frac{\Delta x}{W}\right)^{d}
$$

showing that the computation time is highly sensitive to $\lambda$ and also depends on the spatial resolution required by the shape of the interface profile of $\psi$ : smoother profiles are better!

While it would be computationally efficient to work with large $\lambda$, doing so would introduce higher order terms in the curvature $k$ and velocity $V$ into the GibbsThomson condition (3). In principle, it might be possible to fine tune $g(\psi), f(\psi)$, and $h(\psi)$ to kill terms of order $p^{2}, p q, v^{2}, v q$, etc. However, the resultant expressions are very complicated, the integrals involved cannot be done analytically, there are many terms to consider, and even if successful, this would most likely introduce delicate fine structure into the phase-field profile which would offset the computational benefits.

How can we do better? Using equation (10) we see that we require $v=\left(\tilde{\beta}+a_{2} \lambda\right) p \ll 1$ which puts a very severe constraint on $p$ if $\tilde{\beta}$ is large. Computationally this would be more important at large undercooling when a thin temperature boundary layer forms around the solidification front, and correspondingly $q<p$ so that the smallness of $p$ is the limiting factor. (For example at undercooling $\Delta=0.8$ the theory predicts $q / p=1 / 7$ at a steady state dendrite tip.) Therefore, a good objective is to modify the phase-field in a way that relaxes the constraint on $v$.

A step in that direction was made by Bragard et al. [15], who replaced $\lambda u$ by $H(\lambda u)$ in equation (4). $H(\cdot)$ is computed numerically by solving the following non-linear eigenvalue problem with appropriate boundary conditions on $\psi$ :

$$
\frac{d^{2} \psi}{d x^{2}}-f_{\psi}(\psi)+v \frac{d \psi}{d x}-H(v) g_{\psi}(\psi)=0 .
$$

With $H(\cdot)$ chosen in this way the non-linearities appearing in the standard phase-field model at large $v$ are cancelled by the non-linearities in $H(\cdot)$. To relate the parameters they use

$$
d_{0}=\frac{W}{\lambda}, \beta=\frac{\tau}{\lambda W} .
$$

However, this relationship is valid only in the limit of vanishing $p$, i.e. when $u$ is approximately constant across the diffuse interface - a result analogous to that of Caginalp for the standard phase-field. Correspondingly, in [15] a value of $p$ close to 0.01 is used in computations.

Since $v+q$ is no longer a small parameter it is analytically more involved to derive corrections for finite $p$. To 
compute the linear part it is enough to consider small $v$ and the result is

$$
\tau=\lambda W\left(\beta+a_{2} \frac{W}{D}\right)
$$

which is the analog of Karma and Rappel's formula.

Replacing (14) by (15) allows much larger values of $p$ to be used and is a straightforward way to make better use of the model proposed in [15]. Numerically we observed that for $g_{\psi}=\left(1-\psi^{2}\right)^{2}$, the form used in [15], the non-linearities in $k$ and $V$ are weak even for values of $v$ of the order of 20. However, the Bragard et al. phase-field model, even with the improved asymptotics (15) that we derived, still does not provide the desired degree of computational improvement because the phasefield profile develops a new length scale of order $W / H(v)$ which needs to be resolved numerically in order to avoid artifacts. In addition, $H$ increases very rapidly with $v$.

New class of models:- We propose to replace $\tau$ by $\tau_{R}(\psi)$ in such a way so that the effective equation for $\psi$ becomes

$$
\tau \partial_{t} \psi=W^{2} \nabla^{2} \psi-f_{\psi}(\psi)-\lambda u_{i} W|\nabla \psi|
$$

What are the advantages of doing this? The asymptotic analysis is greatly simplified because the equation for $\psi$ can be analyzed separately from that for $u$. The solution for $\psi$ is simply $\psi(\xi)=\psi_{0}(\xi)$ where $\psi_{0}(\xi)$ is the solution of $\partial_{\xi}^{2} \psi_{0}-f_{\psi}\left(\psi_{0}\right)=0$, and the relation between the parameters is simply given by (14).

Now we can rewrite (16) as

$$
\tau \partial_{t} \psi=W^{2} \nabla^{2} \psi-f_{\psi}-\lambda u W|\nabla \psi|-\lambda \frac{p}{2} F_{1}(\psi) W|\nabla \psi|
$$

with the only problem being the presence of $p$ in the evolution equation. The final trick is to express $p$ in terms of $\partial_{t} \psi$ :

$$
p=\frac{W}{D} V=-\frac{W}{D} \frac{\partial_{t} \psi}{\partial_{x} \psi}=\frac{W}{D} \frac{\partial_{t} \psi}{|\nabla \psi|} .
$$

The equation for $\psi$ becomes

$$
\tau_{R}(\psi) \partial_{t} \psi=W^{2} \nabla^{2} \psi-f_{\psi}(\psi)-\lambda u W|\nabla \psi|,
$$

where

$$
\tau_{R}=\tau-\frac{1}{2} \lambda \frac{W^{2}}{D} F_{1}(\psi)
$$

For $f(\psi)=\frac{1}{4}\left(1-\psi^{2}\right)^{2}$ and $h(\psi)=\psi$ we have $F_{1}(\psi)=$ $\sqrt{2} \ln ((\psi+1) / 2)$. It follows that $\tau_{R} \geq \tau$ which means that the model is well behaved. The expression for $\tau_{R}$ can be compared with Karma and Rappel's formula which can be rewritten in the form $\tau^{\prime}=\tau+a_{2} \lambda \frac{W^{2}}{D} . \quad a_{2}$ is approximately the value of $-1 / 2 F_{1}(0)$. As it stands $\tau_{R}(-1)=\infty$ and points with $\psi=-1$ cannot evolve, so some cutoff near $\psi=-1$ should be introduced. Experiments show that results are insensitive to the exact form of the cutoff.
The restriction of order $p$ accuracy comes from expanding $u(\xi)$ near the interface. It is possible to go to higher orders in $p$ or simply use the full expression (6). This would result in an implicit equation for $\partial_{t} \psi$

$\tau \partial_{t} \psi=W^{2} \nabla^{2} \psi-f_{\psi}-\lambda u W|\nabla \psi|-\frac{\lambda W^{2}}{2 D} \partial_{t} \psi \hat{u}(\psi, p+q)$.

The function $\hat{u}$ can be tabulated in advance and equation (21) can be solved iteratively at each time step.

Different approximations to eqn. (21) lead to different schemes. For example if we consider the next order term in (9) $\frac{1}{2} p(p+q) F_{2}(\psi ;\{\psi(\xi)\})$ we end up with a quadratic equation for $\partial_{t} \psi$. The model including $p^{2}$ corrections is:

$$
\begin{gathered}
\tau_{R}=\tau-\frac{\lambda W^{2}}{2 D}\left(F_{1}(\psi)+q F_{2}(\psi)\right) \\
\tau_{R}\left(\partial_{t} \psi\right)_{0}=W^{2} \nabla^{2} \psi-f_{\psi}(\psi)-\lambda W u|\nabla \psi| \\
\alpha=\left(\partial_{t} \psi\right)_{0} \frac{1}{\tau_{R}} \frac{\lambda W^{4}}{2 D^{2}} \frac{F_{2}(\psi)}{g_{\psi}(\psi)} \\
\partial_{t} \psi=\left(\partial_{t} \psi\right)_{0} \frac{1-\sqrt{1-4 \alpha}}{2 \alpha}=\left(\partial_{t} \psi\right)_{0}\left(1+\alpha+2 \alpha^{2}+\ldots\right)
\end{gathered}
$$

In the evolution equation $q=W k=W \nabla \cdot \mathbf{n}$ with $\mathbf{n}=$ $\nabla \psi /|\nabla \psi|$.

Another application of the technique is to correct for terms of order $p q$ at small undercooling when $p \ll q$. In this regime terms of order $p q$ are not negligible compared to terms of order $p$. To achieve this it is enough to use $\left(\partial_{t} \psi\right)_{0}$ from above without correcting it.

The phase-field model can be generalized to handle arbitrary interface kinetics $u_{i}=-d_{0} k-\mathcal{B}(V)$ at order $p$ and arbitrary $v$. The resultant phase-field model equation is

$$
\begin{aligned}
\tau_{R}(\psi, u) \partial_{t} \psi & =W^{2}\left(\nabla^{2} \psi-k(\nabla \psi \cdot \mathbf{n})\right)-f_{\psi}(\psi) \\
& -\mathcal{B}^{-1}\left(\lambda\left(u+d_{0} k\right)\right) W|\nabla \psi| \\
\tau_{R}(\psi, u) & =\tau-\frac{\lambda W^{2}}{2 D} F_{1}(\psi) \mathcal{B}^{-1^{\prime}}\left(\lambda\left(u+d_{0} k\right)\right)
\end{aligned}
$$

The above recipe for improving phase-field models can be used also in cases when the $\psi$ profile changes with $V$ and $k$. For example it can be applied to the model of Bragard et al. by effectively replacing $u$ with $u_{i}$ yielding

$$
\tau_{R}(\psi, u) \partial_{t} \psi=W^{2} \nabla^{2} \psi-f_{\psi}(\psi)-H(-\lambda u) g_{\psi}(\psi),
$$

with

$$
\tau_{R}=\tau+\frac{\lambda W}{2 D} H^{\prime}(-\lambda u) \tilde{F}_{1}(\psi, H(-\lambda u)) \frac{g_{\psi}(\psi)}{|\nabla \psi|} .
$$

In this case the expression for $\tau_{R}$ is more complicated because $\psi$ changes with $v$. The functions $H(v)$ and $\psi_{v}(\xi)$ which solve equation (13) and $F_{1}$ can be pre-computed numerically leading to a very efficient numerical scheme.

Anisotropy:- To include anisotropy we need only to replace $W$ with $W(\mathbf{n}), \tau$ with $\tau(\mathbf{n})$, and in three dimensions, $W^{2} \nabla^{2} \psi$ with $-\frac{\delta}{\delta \psi} \int d V W(\mathbf{n})^{2}(\nabla \psi)^{2}$ to obtain the Gibbs-Thomson condition

$$
u_{i}=-\frac{1}{\lambda} \sum_{i=1,2}\left[W(\mathbf{n})+\partial_{\theta_{i}}^{2} W(\mathbf{n})\right] \frac{1}{R_{i}}-\frac{\tau(\mathbf{n})}{\lambda W(\mathbf{n})} V,
$$




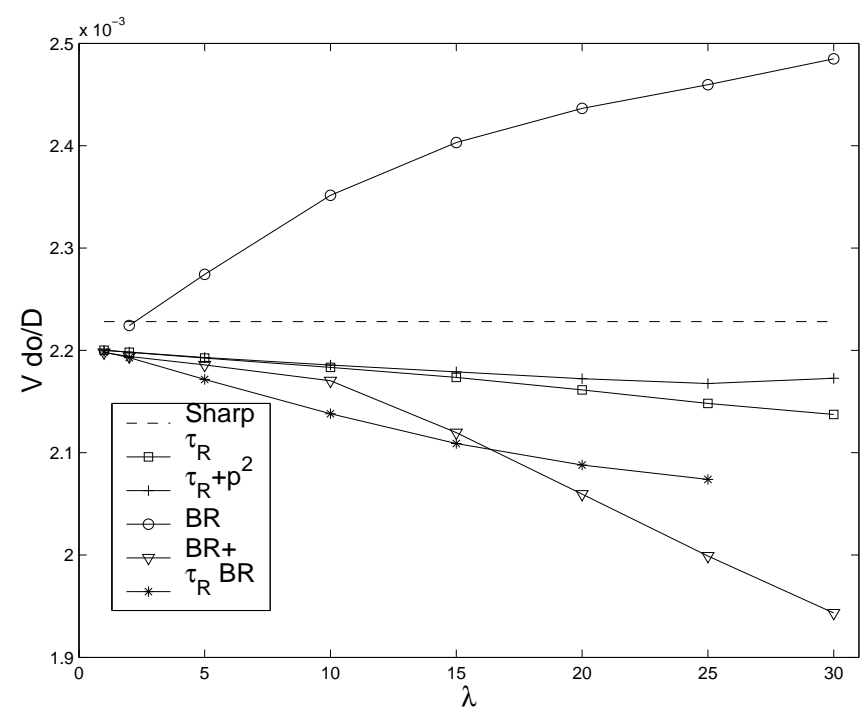

FIG. 1: Interface velocity from different phase-field models as a function of $\lambda$. $\tilde{\beta}=90, \Delta=1.2$. At $t=8 \times 10^{6} d_{0}^{2} / D$. $\Delta x=0.5 W=0.5 \lambda d_{0}$

where $\theta_{1}$ and $\theta_{2}$ are the angles between the normal and the local principle directions on the interface, and $R_{1}^{-1}$ and $R_{2}^{-1}$ - the principle curvatures.

Numerical Experiments:- We now compare the performance of different phase-field models in one-dimensional simulations. The benchmark problem solved is $u(t=$ $0, x)=-\Delta$ for $x \in(-\infty, \infty)$ with the solid-liquid interface initially at $x=0$. The interface velocity, $V(t)$, is compared to that for the sharp interface model obtained via direct numerical integration.

The models compared are identified as follows. $S T$ : standard phase-field model (44); $B R$ : Bragard et al. model with asymptotic relation (14); $B R+$ : the above model with the improved asymptotic relation (15); $\tau_{R}$ : the new model (19); $\tau_{R}+p^{2}$ : the new model with $p^{2}$ cor- rections (22); $\tau_{R} B R$ : the improved version of $B R$ given in (25). We used $h(\psi)=\psi$ and $g_{\psi}=\left(1-\psi^{2}\right)^{2}$.

As an example, we computed the velocity of the front after $t=3.5 \times 10^{4} d_{0}^{2} / D$ for $\tilde{\beta}=10, \Delta=1.2$ and $\lambda=15$. The exact result is $V d_{0} / D=0.021$. The results for models $S T, B R, \tau_{R} B R$ were $0.012,0.044,0.020$ respectively, showing that the previously existing models are inadequate in this regime.

Figure 1 compares the systematic deviations of various phase-field models from the sharp interface solution as a function of $\lambda$. One clearly sees that $B R$ model leads to errors linear in $\lambda$. For $B R+$ unintended nonlinearities in the Gibbs-Thomson condition quickly increase the error with $\lambda$. In contrast $\tau_{R}$ and $\tau_{R}+p^{2}$ models yield approximately the same velocity for the entire range of $\lambda$ considered. Using the values for $\mathrm{Ni}$ cited in [15], $D=10^{-5} \mathrm{~m}^{2} / \mathrm{sec}$ and $d_{0}=5.56 \times 10^{-10}$, the choice $\Delta=1.2$ corresponds to a steady state velocity $V=(\Delta-1) D /\left(\tilde{\beta} d_{0}\right)=40 \mathrm{~m} / \mathrm{sec}$ which is approximately where the experimentally observed morphological transition occurs.

To match the accuracy of $\tau_{R}$ model with $\lambda=30$ we need to take about $\lambda=5$ in $B R$ (measuring deviations from the limiting phase field value). In $3 \mathrm{D}$, this leads to about $(30 / 5)^{3} \approx 200$ times increase in computational speed as compared to the simulations in [15]. If we use the Bragard et al. model with our improved asymptotics, the new $\tau_{R}(\psi)$ models will be about $3^{3}=27$ times faster. The above figures are just for illustration, the precise computational gains will depend on the desired accuracy and the regime of interest.

In conclusion, the models described here are the first that can systematically handle interface kinetics dominated growth in and beyond the thin-interface limit enabling huge gains in computational efficiency.

We thank Jon Dantzig for useful discussions and his interest in this work. This work was supported in part by the National Science Foundation through grants NSFDMR-99-70690 and NSF-DMR-01-21695.
[1] J.S. Langer. In G. Grinstein and G. Mazenko, editors, Directions in condensed matter physics, page 165. World Scientific Press, 1986.

[2] A. Karma and W. Rappel. Phys. Rev. E, 53:R3017, 1996.

[3] N. Provatas, N. Goldenfeld, and J. Dantzig. Phys. Rev. Lett., 80:3308, 1998.

[4] W. Wheeler, G. McFadden, and W. Boettinger. Proc. R. Soc. London A, 452:495, 1996.

[5] G. Caginalp and X. Chen. In M.E.Gurtin and G.B. McFadden, editors, On the evolution of phase boundaries, volume 1, page 1. Springer-Verlag, 1992.

[6] A.Karma and W. Rappel. Phys. Rev. Lett., 77:4050, 1996.

[7] A. Karma and W. Rappel. Phys. Rev. E, 57:4323, 1997.

[8] For a recent review see R. González-Cinca et al. condmat/0305058.
[9] R. Willnecker. Phys. Rev. Lett., 62:2707, 1989.

[10] J. Lum, D. Matson, and M. Flemings. Metall. Mater. Trans. B, 27:865, 1996.

[11] D. M. Matson. In S.P.Marsh, J.A. Dantzig, R. Trivedi, W. Hofmeister, M.G. Chu, E.J. Lavernia, and J. H. Chun, editors, Solidification 1998, volume 1, page 233. The Mineral, Metals and Materials Society, 1998.

[12] W. Hofmeister, R. Bayuzick, and M. Robinson. Rev. Sci. Instrum., 61:2220, 1990.

[13] J. Hoyt, B. Sadigh, M. Asta, and S. Foiles. Acta mater., 47:3181, 1999.

[14] J. Hoyt, M. Asta, and A. Karma. Phys. Rev. Lett., 86:5530, 2001.

[15] J. Bragard, A. Karma, Y. Lee, and M. Plapp. Interface Science, 10:121, 2002. 